\title{
Skin grafting the vascular pedicle: a useful technique to avoid microvascular collapse in free tissue transfer for limb salvage
}

\author{
Alexandra Kovar ${ }^{1}$, Shawn Diamond ${ }^{2}$, Matthew L. Iorio ${ }^{1,2}$ \\ 'Division of Plastic and Reconstructive Surgery, University of Colorado, Anschutz Medical Center, Aurora, CO 80045, USA. \\ 2Beth Israel Deaconess Medical Center, Harvard Medical School Plastic Surgery Residency, Boston, MA 02215, USA.
}

Correspondence to: Dr. Matthew L. Iorio, Division of Plastic and Reconstructive Surgery, University of Colorado, Anschutz Medical Center, Mail Stop C309, 12631 East 17th Ave, Room 6619, Aurora, CO 80045, USA. E-mail: matt.iorio@ucdenver.edu

How to cite this article: Kovar A, Diamond S, lorio ML. Skin grafting the vascular pedicle: a useful technique to avoid microvascular collapse in free tissue transfer for limb salvage. Plast Aesthet Res 2019;6:10. http://dx.doi.org/10.20517/2347-9264.2019.09

Received: 24 Feb 2019 First Decision: 18 Apr 2019 Revised: 1 May 2019 Accepted: 5 May 2019 Published: 23 May 2019

Science Editor: Raúl González-García Copy Editor: Cai-Hong Wang Production Editor: Huan-Liang Wu

\begin{abstract}
Aim: Free tissue transfer is essential for extremity reconstruction following traumatic injuries, oncologic resection, and diabetic complications. However, given the circumferential shape of the arm and leg, a small amount of ongoing edema can prevent a tension-free closure. Additionally, intraoperative thrombosis, vascular disease can lead to proximal exposure of the pedicle or vein grafts. This study evaluates the outcomes of microvascular transfers that utilized a skin graft for closure over the pedicle, in comparison with a matched cohort with a tension-free primary closure.
\end{abstract}

Methods: A retrospective review was completed of all patients that underwent free flap reconstruction of an extremity defect from January 2014 to December 2017 at a single academic institution. Flaps that utilized skin grafting for closure were compared to those closed primarily. Adjunct operative procedures, demographics, and complications were evaluated.

Results: A total of 71 patients fulfilled the inclusion criteria. The $11 \mathrm{flaps}$ in 10 patients underwent skin grafting over the pedicle. The two cohorts were comparable in age, gender, BMI, and co-morbidities, excluding renal disease which was present in $40 \%(n=4)$ of skin grafted group compared to $6.5 \%(n=4)$ in the primary closure group. Flap area, operative time, and anastomosis technique were comparable between the two groups. There was no significant difference in the rates of post-operative complications including partial flap loss, complete flap loss, infection. Mean follow up time in the skin grafting group was 14.2 months and 20.2 months for the primary closure group. 
Conclusion: As per the principal, a tension-free closure is paramount to preventing tissue complications including direct compression of a microvascular pedicle. However, with ongoing tissue edema skin grafting should be considered as a reliable technique to ensure both protection of the pedicle as well as prevention of direct compression without additional complications and comparable post-operative outcomes.

Keywords: Free flap, limb salvage, skin graft, microsurgery

\section{INTRODUCTION}

Extremity reconstruction after trauma, oncologic resection, and diabetic complications often requires free tissue transfer to provide soft tissue coverage of bone, vessel, and nerve. The circumferential shape of the arm and leg, joint surfaces, motion, tendon glide and potential for weight bearing in addition to the relative lack of elasticity of injured soft tissue, provides unique challenges for a tension free closure. Appropriate flap design requires attentive preoperative planning toward the dimension and thickness of a given defect while taking into consideration the anastomotic location, pedicle lie, vector, tension, motion, tendon glide and potential for weight bearing. Even with optimal planning, excess tension placed over a vascular pedicle can lead to flap demise. Tissue edema, ruddiness and tension can impact the survival of free flaps if pressure is applied over anastomoses. Additional factors such as intraoperative thrombosis, pre-existing vascular disease, or other perioperative patient risk factors can lead to proximal exposure of the pedicle or vein grafts $^{[1-3]}$.

Tissue edema, especially secondary to renal disease, can be exacerbated in extremity surgery, secondary to the inflammation of the injury itself, restricted motion, lymphatic disruption or radiation therapy, and tourniquet use ${ }^{[4-7]}$. Unfortunately, these factors can predate the surgery, and in fact represent a contribution to the primary disease state and extremity wound.

With ongoing tissue edema, primary closure after vascular exposure can become increasingly difficult, even to the point of potentially compressing the vascular pedicle or anastomosis. In these cases, the surgeon could choose to mobilize the flap proximally to prevent vascular exposure, but this may leave a portion of the recipient site uncovered. Alternatively, the flap can be left in place as intended to cover the recipient site, and instead, the vascular pedicle is covered with a full or split thickness skin graft. The latter option may prevent desiccation, but it is unclear if skin grafting the anastomosis, vein grafts and pedicle may provoke microvascular collapse.

This study evaluates the outcomes of microvascular transfers that utilized a skin graft for closure over the pedicle, in comparison with a matched cohort that achieved primary closure. The authors hypothesized that skin grafting provides a tension-free closure when primary closure is unable to be performed and can safely salvage a free-flap reconstruction without an increase in flap related or patient morbidity.

\section{METHODS}

All extremity free flaps performed at a single, Level 1 trauma center were entered into a prospectively maintained registry including patient demographic information, clinical history, radiographic imaging, procedural data, operative reports, postoperative care and long-term complications across 118 unique variables. A REDCap database was utilized as a secure web-based application for data maintenance. A trained member of the research team uploads data once monthly. Follow up clinic visits and photography are specifically analyzed to identify limb salvage, flap failure, wound recurrence, patient ambulation, use of assistive devices, patient disposition and rates of amputation. The database is maintained via institutional review board approval. Operative reports were queried specifically wherein description of skin grafts placed 
directly over the pedicle were separated from those reports with skin grafting elsewhere such as the donor site or atop the flap.

For the purposes of this study, the database was queried in October 2018 for cases performed from January 2014 to December 2017. For each patient, relevant demographic information, comorbidities, presence of chronic kidney disease, arterial revascularization, anticoagulant use, wound etiology, pre-operative imaging, anatomical wound location, skeletal fixation, flap thickness, operative characteristics, complications and follow-up were reviewed.

Guiding principles in lower extremity reconstruction were followed: appropriate debridement to perfused tissue, preservation of vital structure, muscle, nerve and tendon along with isolation and control of major vascular inflow. Wounds amenable to local tissue reconstruction with advancement flaps, skin-graft, regional pedicle flaps, freestyle propeller flaps were utilized when-able but excluded from this study.

During free tissue transfer, we preferentially performed end-end anastomosis in patients with adequate runoff. However, in settings of critical limb ischemia or compromised in-flow an end to side anastomosis was performed. We have previously studied an algorithm for venous anastomosis and preferentially utilize the deep venous system, avoiding refluxing veins and matching for size ${ }^{[8]}$.

An enhanced recovery protocol was utilized for the majority of our patients including the use of regional anesthetic block $^{[9]}$ and an early limb dependency program ${ }^{[10]}$ helped patients dangle early in their postoperative course expediting hospital stay, discharge to rehabilitation facilities, and return toward functional ambulation.

Primary closure over the pedicle was defined as direct closure of at least the skin layer with tissue from the recipient site, or in combination with a portion of the flap. Tension was evaluated by the inability to close the skin and or skin-flap interface with a 3-0 nylon and a double-knot throw, without slipping. Skin grafting closure required a separate donor site for harvesting the skin graft to place over the fasciocutaneous free flap to provide an additional layer of coverage. Often, in the case of anterolateral thigh free flaps - we were able to utilize the dog-ears from the apices of the lateral thigh incision to create a full thickness skin graft in cases of small $(<6 \mathrm{~cm} \times 6 \mathrm{~cm})$ areas of pedicle exposure. For any larger dimensions a dermatome was used at 1/14,000 of an inch to place a split thickness skin graft over the pedicle. Thorough attention toward dressing and splinting the extremity was performed. Xeroform (Covidien, Dublin, Ireland) was placed over the skin-graft and pedicle construct without a bolster or pressure dressing. The flap and extremity were wrapped in bulky jones cotton, a plaster splint and ACE to ensure appropriate padding and pressure offloading of both flap and pedicle. We monitored the flaps using clinical exam, Doppler probes and Vioptix (ViOptix Inc. Newark, CA).

\section{Outcome measures}

Outcomes pertaining to flap specific morbidity such as partial flap loss, microvascular collapse, vessel thrombosis, site infection and dehiscence were analyzed in addition to systemic complications as well as need for operative take-back. Additionally, vascular pedicle exposure and loss of the skin graft were also analyzed.

\section{Analysis}

Descriptive statistics were utilized to compare patient demographic information in regard to number, frequency, mean and standard deviation. Student $t$-test for continuous data and Fischer's exact test for categorical data were used for univariate analysis to determine significant differences between skin graft and primary closure groups. Those variables achieving significance $P<0.05$ were entered into a multivariable 
Table 1. Patient and wound characteristics compared between patients with vascular pedicle skin grafting and primary closure

\begin{tabular}{|c|c|c|c|c|}
\hline Variable & Skin graft to pedicle $(n=13)$ & Pedicle closed $(n=63)$ & Odds ratio & $P$ value \\
\hline \multicolumn{5}{|l|}{ Demographics } \\
\hline AGE Mean (Range) & $47(47-69)$ & $59(20-77)$ & - & $>0.05$ \\
\hline Male & $8(61 \%)$ & $48(76 \%)$ & 0.41 & 0.21 \\
\hline \multicolumn{5}{|l|}{ Comorbidity } \\
\hline BMI & $28(21-40)$ & $27(19-44)$ & - & $>0.05$ \\
\hline Diabetes & $7(54 \%)$ & $18(28 \%)$ & 2.3 & 0.21 \\
\hline Malnourished albumin $>3$ & $7(54 \%)$ & $10(16 \%)$ & 5.1 & 0.02 \\
\hline Renal disease CKD & $6(46 \%)$ & $4(6.3 \%)$ & 9.5 & 0.0065 \\
\hline Tobacco & $2(15 \%)$ & $25(39.6 \%)$ & 0.16 & 0.09 \\
\hline Peripheral arterial disease & $4(31 \%)$ & $13(20.6 \%)$ & 1.6 & 0.54 \\
\hline Coronary artery disease & $1(7.7 \%)$ & $7(11.1 \%)$ & 0.86 & 0.89 \\
\hline Preop antiplatelet agent (ASA/Plavix) & $5(38 \%)$ & $43(68.3 \%)$ & 0.41 & 0.21 \\
\hline Preop vascular imaging (CTA/Angio) & $6(46 \%)$ & $33(52.3 \%)$ & 1.27 & 0.72 \\
\hline \multicolumn{5}{|l|}{ Recipient wound } \\
\hline Pre-flap vascular intervention & $2(15 \%)$ & $7(11.1 \%)$ & 1.9 & 0.45 \\
\hline Peripheral bypass & $0(0 \%)$ & $2(3.1 \%)$ & - & - \\
\hline Endo vascular revascularization & $2(15 \%)$ & $6(9.5 \%)$ & 2.3 & 0.36 \\
\hline Procedure done & Aplasty, bypass & Aplasty, bypass & & \\
\hline$<3$ Vessel runoff & $7(54 \%)$ & $16(25.4 \%)$ & 6.56 & 0.01 \\
\hline Upper extremity & $1(7 \%)$ & $7(11.1 \%)$ & - & - \\
\hline Lower extremity & $12(93 \%)$ & $54(85 \%)$ & 1.56 & 0.69 \\
\hline Previous amputation & $4(31 \%)$ & $8(12.7 \%)$ & 4.42 & 0.04 \\
\hline \multicolumn{5}{|l|}{ Wound etiology } \\
\hline Arterial & $6(46 \%)$ & $27(42.8 \%)$ & 1.89 & 0.36 \\
\hline Traumatic & $5(38 \%)$ & $35(55.5 \%)$ & 0.49 & 0.31 \\
\hline Malignant & $2(15 \%)$ & $8(12.7 \%)$ & 1.65 & 0.56 \\
\hline Chronic wound & $4(31 \%)$ & $25(39.6 \%)$ & 0.96 & 0.95 \\
\hline Infected & $5(38 \%)$ & $37(58.7 \%)$ & 0.64 & 0.52 \\
\hline Hardware & $2(15 \%)$ & $21(33.3 \%)$ & 0.47 & 0.37 \\
\hline Osteomyelitis proven & $3(23 \%)$ & $17(26.9 \%)$ & 1.1 & 0.89 \\
\hline Revascularization & $2(15 \%)$ & $6(9.5 \%)$ & 2.29 & 0.35 \\
\hline
\end{tabular}

The table compares patients with pedicle skin-grafting versus primary closure over the pedicle. Patient demographic features, comorbidities, wound etiology and location are described by rate and frequency. Univariate analysis was performed and reported as odds ratios where appropriate. $P<0.05$ are highlighted and reached statistical significance

regression model to identify independent risk factors associated with flap loss. Statistical significance was defined as a $P<0.05$. All analyses were performed using Prism, version 5.0b (GraphPad Software, La Jolla, CA).

\section{RESULTS}

The review identified 75 patients who underwent a total of 76 fasciocutaneous free flaps with either a primary closure over the vascular pedicle $(n=63)$ or a skin graft $(n=13)$ closure [Table 1].

Patient comorbidities included diabetes $(n=25)$, chronic kidney disease $(n=10)$, tobacco use $(n=27)$, peripheral vascular disease $(n=17)$, and coronary artery disease $(n=8)$. Additional clinical characteristics, such as antiplatelet use, were assessed as contributing factors to wound etiology. The two cohorts were comparable in age, gender, BMI, and co-morbidities, excluding renal disease which was present in $46 \%$ $(n=6)$ of skin grafted group compared to $6.3 \%(n=4)$ in the primary closure group. The pathogenesis of all patient wounds included history of trauma $(n=40)$, malignancy $(n=10)$, chronic wounds $(n=29)$, infected wounds $(n=42)$, and hardware exposure requiring tissue coverage $(n=23)$.

Skin grafting closure was performed for 13 flaps, while the remaining 63 flaps utilized primary closure. The difference in flap area was not significantly different between the skin graft and primary closure groups, 
Table 2. Surgical details compared between groups of vascular pedicle closure

\begin{tabular}{|c|c|c|c|c|}
\hline Surgical details & Skin graft to pedicle $(n=13)$ & Pedicle covered $(n=63)$ & Odds ratio & P value \\
\hline \multicolumn{5}{|l|}{ Operative details } \\
\hline Single vein & $4(31 \%)$ & $24(38.1 \%)$ & 1 & 0.96 \\
\hline Dual venous outflow & $6(46 \%)$ & $37(58.7 \%)$ & 0.97 & 0.97 \\
\hline End-to-end & $11(85 \%)$ & $58(92 \%)$ & - & $>0.05$ \\
\hline End-to-side & $2(15 \%)$ & $3(4.8 \%)$ & - & $>0.05$ \\
\hline Coupler sizes mode (Min-Max) mm & $2.0 \mathrm{~mm}(1.5-2.5 \mathrm{~mm})$ & $2.5 \mathrm{~mm}(1.5-3.5 \mathrm{~mm})$ & - & $>0.05$ \\
\hline Flap area (Avg) & $17.3 \mathrm{~cm} \times 8.4 \mathrm{~cm}$ & $19.1 \mathrm{~cm} \times 8.2 \mathrm{~cm}$ & - & $>0.05$ \\
\hline OR time (Minimun) & $420 \min (340)$ & $445 \min (180)$ & - & $>0.05$ \\
\hline
\end{tabular}

$145.3 \mathrm{~cm}^{2}$ and $156.6 \mathrm{~cm}^{2}$ respectively. OR time was also similar between the two groups with $420 \mathrm{~min}$ in the skin graft group and $445 \mathrm{~min}$ in the primary closure group. Arterial anastomoses were done in an endto end fashion in $82 \%(n=11)$ skin grafting cases and $92 \%(n=58)$ of primary closure cases. Dual venous outflow was used to drain the flaps in $46 \%$ of the skin grafted group and $58.7 \%$ of the primary closure group $(n=6, n=37)$. Operative details are described in Table 2.

Mean follow up time in the skin grafting group was 16.2 months and 22.2 months for the primary closure group. Flap complications occurred in 23 cases $(n=4, n=19)$. Individual patient complications are outlined in Table 3.

Total flap loss occurred in 4 primary closure flaps and 1 skin grafted flap. Partial flap loss occurred in 3 primary closure flaps and only 1 skin grafted flap. The most common risk factors among patients with flap loss were pre-existing vascular disease and pre-operative endovascular interventions. In multivariable regression, preoperative arterial revascularization was the only factor identified as associated independently with flap loss. There were no instances of arterial thrombosis or insufficiency in the skin grafted group. There was no statistically significant difference between rates of post-operative complications between the two groups including partial flap loss, complete flap loss, arterial inflow or outflow complications, flap infection, and dehiscence. No flap removals occurred in the skin grafted cohort compared to $7.9 \%(n=5)$ in the primary closure group.

\section{DISCUSSION}

The results of this study demonstrate that skin graft closure over a microvascular pedicle can be performed without an increased rate of microvascular complications. Grafting over the pedicle is a safe alternative to primary closure when undue tension may create a risk for vascular compression. There were no cases of arterial thrombosis or insufficiency in the skin grafted group that is likely due to the lack of pressure from a tight, primary closure. There were no statistically significant differences in the post-operative complications between the two groups, no flaps in the skin grafted group required flap removal where there were 5 cases in the primary closure group.

To date, one article was identified in the literature documenting a single case report of primary skin grafting over the vascular pedicle leading to flap salvage ${ }^{[11]}$. However, this technique is performed routinely at our institution. The venous flow-through flap is reported with greater frequency in digital and upper extremity replantation/revascularization literature ${ }^{[12]}$ particularly for coverage of volar soft tissue defects with exposure of digital vessels in ring avulsions, or amputations with soft tissue destruction. However, full thickness skin grafts have also been used in this setting and reported.

Literature regarding flap complications is heterogeneous and limited to retrospective analysis of perioperative patient factors ${ }^{[3,13,14]}$. Diabetes, tobacco use, long operating times have all been identified as contributing to flap demise. Diabetes negatively affects the microvasculature causing changes in 
Table 3. Flap related complications compared between groups by vascular pedicle coverage

\begin{tabular}{|c|c|c|c|c|}
\hline Complication & Skin graft to pedicle $(n=13)$ & Pedicle covered $(n=63)$ & Odds ratio & $P$ value \\
\hline \multicolumn{5}{|l|}{ Flap outcomes } \\
\hline Any flap complication & $4(31 \%)$ & $19(30.1 \%)$ & 1.7 & 0.44 \\
\hline Early than 7 days take back & $2(15 \%)$ & $8(12.6 \%)$ & 1.9 & 0.46 \\
\hline Later than 7 days surgery & $1(7 \%)$ & $9(14.2 \%)$ & 0.64 & 0.69 \\
\hline Venous thrombosis & $2(15 \%)$ & $5(7.9 \%)$ & 4.8 & 0.11 \\
\hline Arterial thrombosis & $0(0 \%)$ & $3(4.7 \%)$ & - & - \\
\hline Arterial insufficiency & $0(0 \%)$ & $1(1.6 \%)$ & - & - \\
\hline Partial flap loss skin soft tissue fascia & $1(7 \%)$ & $3(4.7 \%)$ & 3.3 & 0.34 \\
\hline Complete flap loss & $1(7 \%)$ & $4(6.3 \%)$ & 1.58 & 0.69 \\
\hline Flap infection & $0(0 \%)$ & $4(6.3 \%)$ & - & - \\
\hline Dehiscence & $2(15 \%)$ & $4(6.3 \%)$ & 3.56 & 0.11 \\
\hline Contour surface irregularity & $0(0 \%)$ & $0(0 \%)$ & - & - \\
\hline Debulking & $1(7 \%)$ & $2(3.2 \%)$ & 3.2 & 0.35 \\
\hline Flap elevation & $1(7 \%)$ & $9(14.3 \%)$ & 0.64 & 0.69 \\
\hline Flap removal & $0(0 \%)$ & $5(7.9 \%)$ & - & - \\
\hline Amputation & $0(0 \%)$ & $2(3.1 \%)$ & - & - \\
\hline Follow up average & 16.2 months & 22.21 months & & $P>0.05$ \\
\hline
\end{tabular}

compliance, elasticity, and ultimately the blood flow through the vessel. These damaged vessels are used for the anastomosis in a free flap ${ }^{[15-17]}$. Lee et al. ${ }^{[15]}$ demonstrated that patients with serum Creatinine greater than $1.28 \mathrm{mg} / \mathrm{dL}$ had significantly higher post-operative complication rates including, partial and total necrosis. Flap reconstruction to previously radiated tissues has been shown to have complication rates between $8 \%-39 \%$, likely due to reduction in vascularization and mean capillary lumen ${ }^{[18,19]}$. Utilizing surgical techniques to minimize the tension, pressure, and manipulation of these vessels is imperative for flap survival.

Limb salvage techniques are utilizing thinner flap techniques more frequently as outcomes are proving to be equivalent to the standard anterolateral thigh (ALT) flap with the added benefit of reduced re-operations and revisions for debulking, as seen in Figure $1^{[20,21]}$. In a series of 25 super-thin or supra-fascial ALT flaps, Seth et al ${ }^{[21]}$ had no episodes of partial or total flap losses. In another study by Hong et al. ${ }^{[7]}$, a flap survival rate of $98 \%$ was demonstrated with the super-thin ALT technique. Dr. Hong ${ }^{[16]}$, like our group, identified preoperative revascularization as an independent risk factor toward flap loss even when utilizing supermicrosurgery and outflow preserving techniques.

Primary closure of a fasciocutaneous flap, as in Figure 2, can be difficult when the original defect's contour has changed due to long OR exposure, fluid resuscitation, and tissue manipulation. There is no current literature evaluating alternative techniques. This study demonstrates skin grafting as a method for flap closure that will minimize risk of vascular compromise and maintain the preoperative dimensions of the flap.

The retrospective design of this study limits the scope of the data that is obtained and potential for randomization. Our series consists of 75 patients over a period of 36 months. Although this is a small volume study of 76 flaps, this is the first series to evaluate skin graft closure. A limitation of this study is the difference between the two cohorts and co-morbid conditions. Patients in the skin grafted group had increased rates of peripheral vascular disease, renal disease, and history of amputations. These specific comorbid conditions are associated with increased post-operative complication rates; however, our study did not demonstrate this. This study found comparable outcomes between skin graft closure and primary closure in a higher risk cohort, demonstrating another indication for this surgical technique. A prospective, randomized study aimed at evaluating closure techniques and the clinical outcomes is necessary.

In conclusion, skin graft closure over free flap anastomoses and pedicles may be considered a safe, alternative technique to prevent compression of the microvascular pedicle for extremity free tissue transfers. Skin grafting 

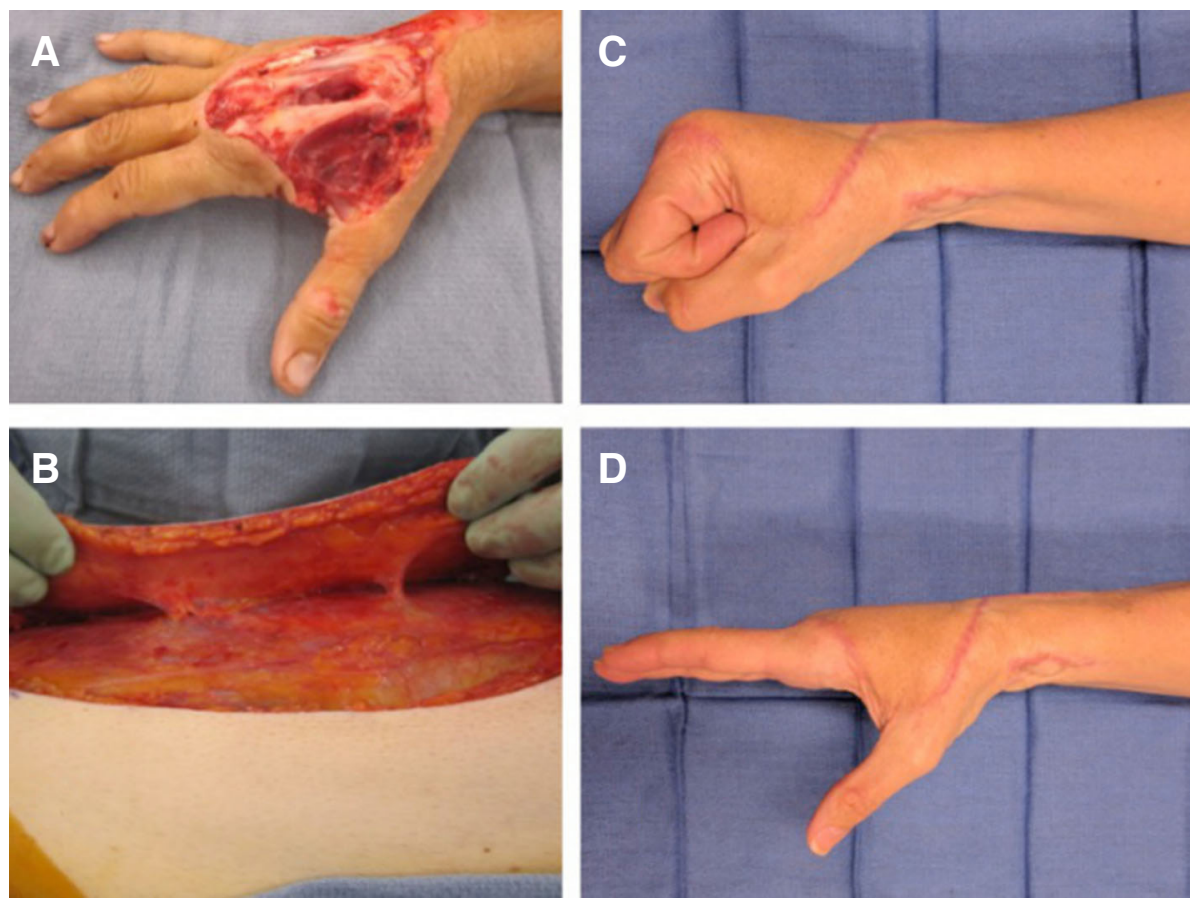

Figure 1. Right hand suprafascial anterolateral thigh (ALT) coverage with skin graft over radial artery. A patient with dorsal hand degloving and exposed extensor tendon in zones 5, 6,7 (A) preoperative photograph after debridement; (B) shows a suprafascial ALT to match the defect thickness, allow tendon glide and two-stage tendon reconstruction with the ability to elevate. The ALT pedicle was tunneled in the region of the anatomic snuffbox. The LFCA was anastomosed to the radial artery at the level of the wrist. A small full thickness skin graft was used to cover the anastomosis and came from the dog-ear of the lateral thigh donor; Postoperative photos after tendon reconstruction show restoration of hand function (C) (D) and a low profile skin graft at the wrist

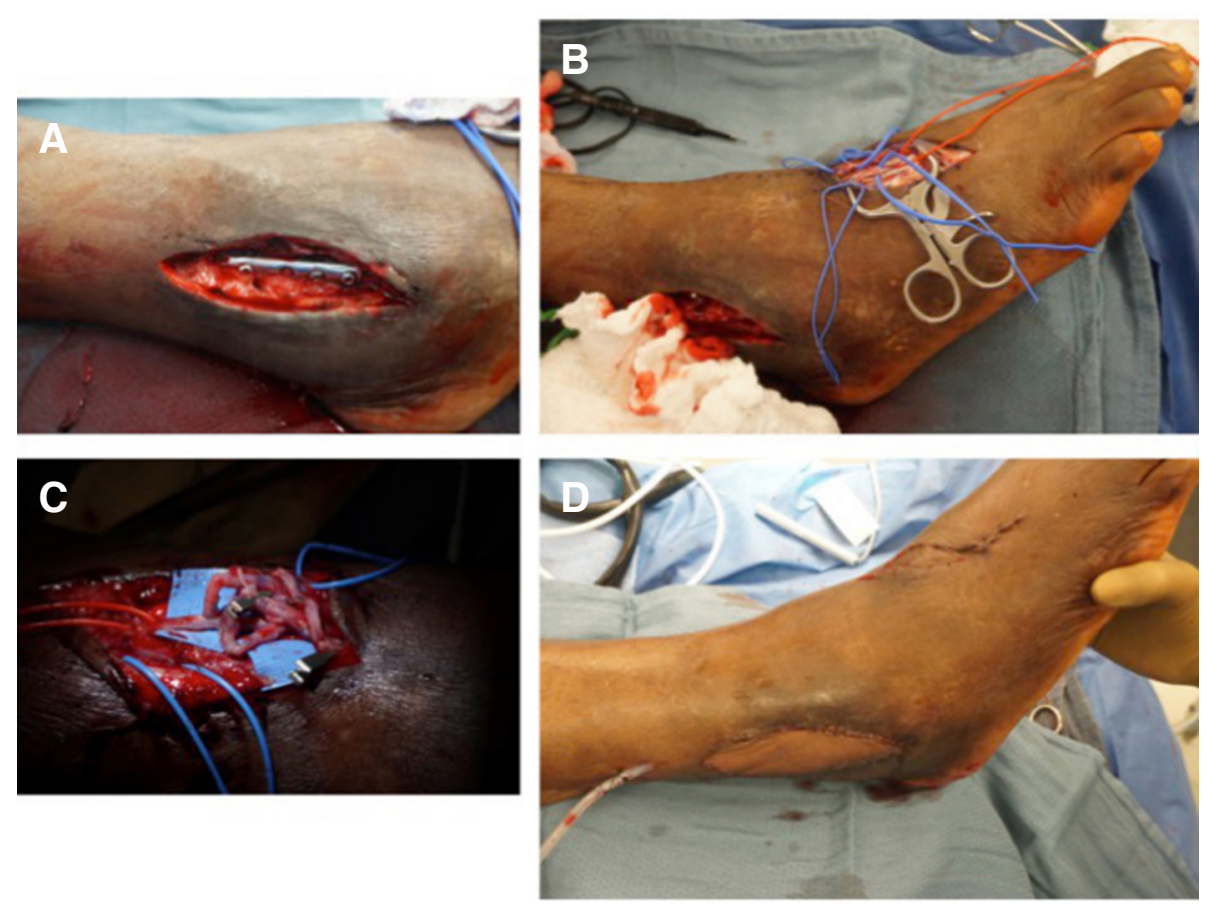

Figure 2. Exposed hardware and fibular non-union - radial forearm flap with dorsalis pedis skin graftA patient with (A) exposed hardware over a fibula fracture at ankle mortis; (B) after exposure of the dorsalis pedis artery, venae comitants and saphenous vein; (C) showing the vascular anastomosis of a single artery end-side, two venae comitants of the radial artery system to dorsalis pedis and cephalic to saphenous anastomosis; (D) this created a bulky vascular group ultimately covered with a split thickness skin graft. The small $3 \mathrm{~cm} \times 1 \mathrm{~cm}$ skin graft donor site was closed primarily 
ensures both protection of the pedicle as well as prevention of direct compression as well as dessication. This technique should be applied to flaps at high risk for major complication, including those exposed to long operative times, pre-existing vascular disease, and flaps requiring anastomotic revisions.

\section{DECLARATIONS}

\section{Authors' contributions}

Concept and design: Iorio ML

Data acquisition and analysis: Diamond S

Manuscript preparation: Kovar A

Critical revision and completion of manuscript: Kovar A, Diamond S, Iorio ML

\section{Availability of data and materials}

Data were strictly obtained from medical records, in accordance with the privacy policy and code of ethics at our institutions.

\section{Financial support and sponsorship}

None.

\section{Conflicts of interest}

All authors declare that there are no conflicts of interest.

\section{Ethical approval and consent to participate}

The study was approved by the BIDMC Institutional Review Board (2017-P-000253).

\section{Consent for publication}

Not applicable.

\section{Copyright}

(c) The Author(s) 2019.

\section{REFERENCES}

1. Pohlenz P, Blessmann M, Blake F, Li L, Schmelzle R, et al. Outcome and complications of 540 microvascular free flaps: the Hamburg experience. Clin Oral Investig 2007;11:89-92.

2. Preidl RH, Wehrhan F, Schlittenbauer T, Neukam FW, Stockmann P. Perioperative factors that influence the outcome of microsurgical reconstructions in craniomaxillofacial surgery. Br J Oral Maxillofac Surg 2015;53:533-7.

3. Gedebou TM, Wei FC, Lin CH. Clinical experience of 1284 free anterolateral thigh flaps. Handchir Mikrochir Plast Chir 2002;34:239-44.

4. Li RG, Ren GH, Tan XJ, Yu B, Hu JJ. Free flap transplantation combined with skin grafting and vacuum sealing drainage for repair of circumferential or sub-circumferential soft-tissue wounds of the lower leg. Med Sci Monit 2013;19:510-7.

5. Eisenhardt SU, Schmidt Y, Thiele JR, Iblher N, Penna V, et al. Negative pressure wound therapy reduces the ischaemia/reperfusionassociated inflammatory response in free muscle flaps. J Plast Reconstr Aesthet Surg 2012;65:640-9.

6. Hong JP. Reconstruction of the diabetic foot using the anterolateral thigh perforator flap. Plast Reconstr Surg 2006;117:1599-608.

7. Hong JP, Chung IW. The superficial fascia as a new plane of elevation for anterolateral thigh flaps. Ann Plast Surg 2013;70:192-5.

8. Mattos D, Diamond S, Chattha AS, Riesel JN, Iorio ML. Venous anastomoses in anterolateral thigh flaps for the lower extremity: vessel selection in lieu of obligatory number. Ann Plast Surg 2018; doi: 10.1097/SAP.0000000000001431.

9. Ruan QZ, Diamond S, Zimmer S, Iorio ML. Assessing the safety and efficacy of regional anesthesia for lower extremity microvascular reconstruction: enhancing recovery. J Reconstr microsurg 2018;34:293-9.

10. Seth AK, Diamond S, Iorio ML. Outcomes of an early protocol for dependent conditioning in lower extremity microsurgical free flaps. J Reconstr Microsurg 2017;33:670-8.

11. Thione A, Cavadas PC, Landin L, Ibañez J. Microvascular pedicle coverage with split thickness skin graft: Indications and surgical tips. Indian J Plast Surg 2011;44:528-9.

12. Brandt K, Khouri RK, Upton J. Free flaps as flow-through vascular conduits for simultaneous coverage and revascularization of the hand or digit. Plast Reconstr Surg 1996;98:321-7. 
13. Adler SS, Afanasiev S, Aidala C, Ajitanand NN, Akiba Y, et al. Measurement of transverse single-spin asymmetries for midrapidity production of neutral pions and charged hadrons in polarized $p+p$ collisions at square root(s) $=200 \mathrm{GeV}$. Phys Rev Lett 2005;95:202001.

14. Kwok AC, Agarwal JP. An analysis of free flap failure using the ACS NSQIP database. Does flap site and flap type matter? Microsurgery 2017;37:531-8.

15. Lee YK, Park KY, Koo YT, Baek RM, Heo CY, et al. Analysis of multiple risk factors affecting the result of free flap transfer for necrotising soft tissue defects of the lower extremities in patients with type 2 diabetes mellitus. J Plast Reconstr Aesthet Surg 2014;67:624-8.

16. Hong JPJ, Goh TLH, Choi DH, Kim JJ, Suh HS. The efficacy of perforator flaps in the treatment of chronic osteomyelitis. Plast Reconstr Surg 2017;140:179-88.

17. Suh HS, Oh TS, Lee HS, Lee SH, Cho YP, et al. A new approach for reconstruction of diabetic foot wounds using the angiosome and supermicrosurgery concept. Plast Reconstr Surg 2016;138:702e-9e.

18. Schultze-Mosgau S, Grabenbauer GG, Radespiel-Tröger M, Wiltfang J, Ries J, et al. Vascularization in the transition area between free grafted soft tissues and pre-irradiated graft bed tissues following preoperative radiotherapy in the head and neck region. Head Neck 2002;24:42-51.

19. Wehrhan F, Rödel F, Grabenbauer GG, Amann K, Brückl W, et al. Transforming growth factor beta 1 dependent regulation of Tenascin-C in radiation impaired wound healing. Radiother Oncol 2004;72:297-303.

20. Diamond S, Seth AK, Chattha AS, Iorio ML. Outcomes of subfascial, suprafascial, and super-thin anterolateral thigh flaps: tailoring thickness without added morbidity. J Reconstr Microsurg 2018;34:176-84.

21. Seth AK, Iorio ML. Super-thin and suprafascial anterolateral thigh perforator flaps for extremity reconstruction. J Reconstr Microsurg 2017;33:466-73. 